\title{
Optimization of Variable-Depth Liner Configurations for Increased Broadband Noise Reduction
}

\author{
M. G. Jones; W. R. Watson† D. M. Nark $\ddagger$ and N. H. Schiller ${ }^{\S}$ \\ NASA Langley Research Center, Hampton, VA 23681 \\ and \\ J. C. Born $\llbracket$ \\ Northrop Grumman Corporation, Hampton, VA 23681
}

\begin{abstract}
This paper employs three acoustic propagation codes to explore variable-depth liner configurations for the NASA Langley Grazing Flow Impedance Tube (GFIT). The initial study demonstrates that a variable impedance can acceptably be treated as a uniform impedance if the spatial extent over which this variable impedance occurs is less than one-third of a wavelength of the incident sound. A constrained optimization study is used to design a variable-depth liner and to select an optimization metric. It also provides insight regarding how much attenuation can be achieved with variable-depth liners. Another optimization study is used to design a liner with much finer chamber depth resolution for the Mach 0.0 and 0.3 test conditions. Two liners are designed based on spatial rearrangement of chambers from this liner to determine whether the order is critical. Propagation code predictions suggest this is not the case. Both liners are fabricated via additive manufacturing and tested in the GFIT for the Mach 0.0 condition. Predicted and measured attenuations compare favorably across the full frequency range. These results clearly suggest that the chambers can be arranged in any order, thus offering the potential for innovative liner designs to minimize depth and weight.
\end{abstract}

\section{Nomenclature}

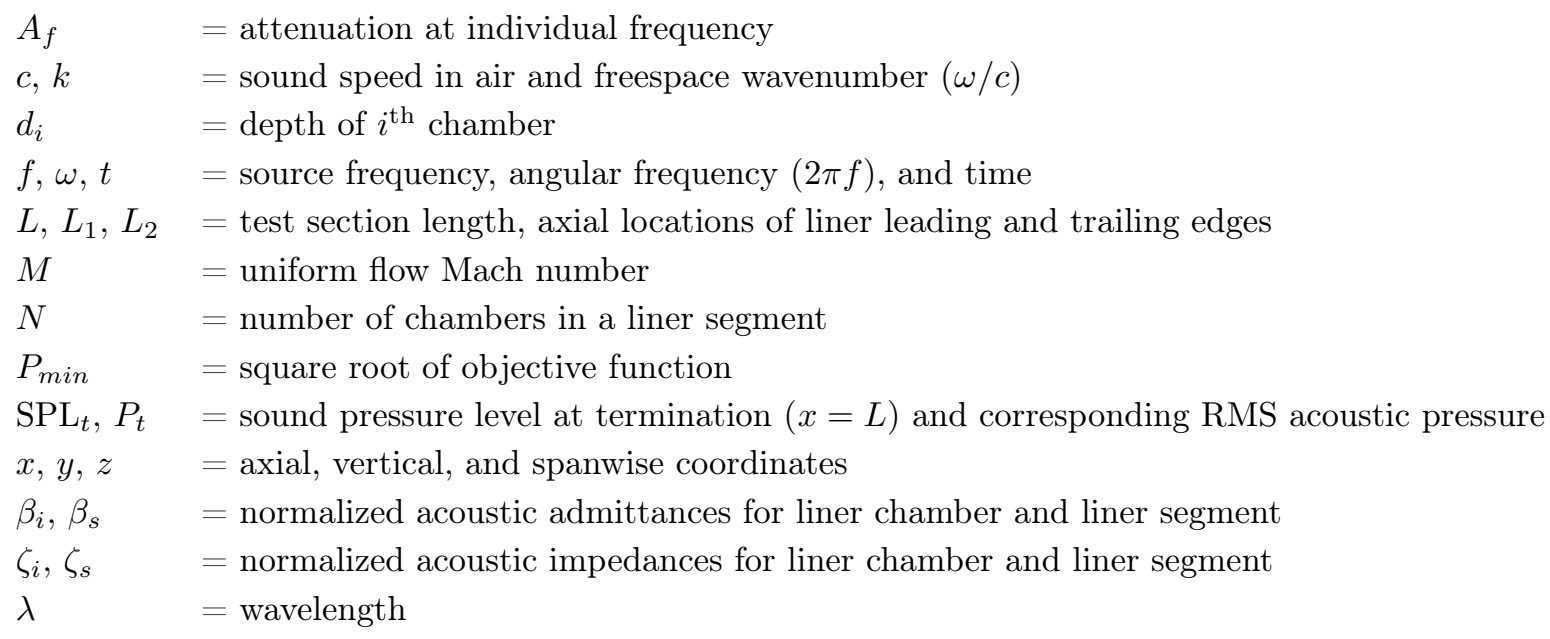

${ }^{*}$ Senior Research Scientist, Research Directorate, Structural Acoustics Branch, AIAA Associate Fellow.

†Senior Research Scientist, Research Directorate, Computational AeroSciences Branch, AIAA Associate Fellow.

¥Senior Research Scientist, Research Directorate, Structural Acoustics Branch, AIAA Associate Fellow.

$\S$ Research Scientist, Research Directorate, Structural Acoustics Branch.

TResearch Engineer. 


\section{Introduction}

Although commercial air traffic continues to increase, there is significant impetus for further reductions in aircraft noise exposure for communities surrounding airports. This has caused the Federal Aviation Administration (FAA) and International Civil Aviation Organization (ICAO) to impose increasingly stringent aircraft noise requirements. In response to these requirements, one of the key components of NASA's Advanced Air Vehicles Program is to develop innovative aircraft noise reduction concepts. One concept that is used for reduction of noise attributed to the fan is to embed acoustic liners in the interior walls of the aircraft engine nacelles. Historically, these liners have been designed for reduction of fan noise dominated by tones (e.g., at multiples of the blade passage frequency). However, a steady increase in the bypass ratio of commercial aircraft engines has caused the dominant noise to become more broadband. Thus, the focus for acoustic liners has also transitioned toward broadband fan noise reduction.

Significant broadband noise reduction has been achieved via multi-degree-of-freedom (MDOF) approaches. One approach that is widely used is to replace single-layer, perforate-over-honeycomb liners with double-layer liners with embedded porous septa (these septa can be implemented in different ways). A second approach is to use different single-layer liners in the inner and outer walls of the aft fan duct. In this latter approach, the inner and outer wall liners are tuned to different frequencies, and thus can be combined to achieve noise reduction over a wider frequency range. For many engine nacelle designs, these approaches are clearly sufficient to effectively reduce the level of radiated noise. However, there remains a need for additional broadband noise reduction, to include (1) an extension of the frequency range over which noise reduction occurs, and (2) an increase in the amount of noise reduction achieved. One concept that offers potential for achieving this increased broadband noise reduction is the variable-depth liner (liner depth varies in axial and/or circumferential directions). ${ }^{1,2}$ This concept is also of interest because of practical concerns. Because of the limited volume within the engine nacelle walls, support hardware (e.g., wiring, air-conditioning ductwork) often infringes on the space desired for installation of the acoustic liners, such that the available depth of the liners varies along the length of the nacelle. Hence, it is important to better understand the effects of axially variable-depth liners.

This variable-depth concept has been a recent focus for the NASA Langley Research Center Liner Physics Team. The first study ${ }^{1}$ compared results achieved with variable-depth liners constructed with ceramic matrix composite (CMC) and stereolithography (SLA). The focus of that study was to evaluate the effects of material porosity for the CMC liner, to determine whether the acoustic performance of this liner could be predicted with conventional models. The interest in CMC material was based on a desire to develop broadband liners for use in the engine core, where the temperatures are quite high. This study demonstrated the suitability of the CMC material (at least from an acoustic standpoint) for this application, and also demonstrated that significant broadband absorption may be achievable via axially variable-depth liners.

The second study ${ }^{2}$ provided a more detailed evaluation of two SLA, variable-depth liners based on tests conducted in the NASA Langley Grazing Flow Impedance Tube (GFIT). This study demonstrated that the sound propagation through the GFIT could be predicted quite well via two propagation codes based on the solution to the convected Helmholtz equation. The CHE $\operatorname{code}^{3}$ (sometimes labeled as Q3D-FEM in earlier papers) employs a finite element method to solve the convected Helmholtz equation, whereas the CDL $\operatorname{code}^{4}$ (or CDUCT-LaRC) utilizes a parabolic approximation to this equation to improve the computational efficiency and to support application to three-dimensional geometries. This study also provided a series of simulations using the $\mathrm{CHE}$ code, in which multiple variable-depth configurations were considered. The results confirmed that axially variable-depth liners can be used to achieve significant broadband noise reduction. It was also observed that this noise reduction was relatively independent of the spatial sequence in which the variable-depth chambers were placed. If this finding could be demonstrated to be universal, i.e., to apply regardless of the acoustic environment (source) or geometry of the duct, it would offer significant potential to liner manufacturers. Based on results presented by Howerton et al., ${ }^{5,6}$ the chambers that form the acoustic liner are not required to be straight and perpendicular to the surface of the liner. Instead, these chambers can be bent (e.g., to form ' $L$ '- and ' $J$ '-shaped chambers) with limited, if any, change in acoustic performance. Thus, a suitable combination of short and long (but bent) chambers could be used to achieve a broadband liner with a reduced liner thickness.

A few key questions remain regarding the suitability of variable-depth liners for use in aft bypass ducts. First, were the conclusions of the earlier computational study limited to the particular configurations chosen, 
or can they be assumed to hold for any variable-depth liner? Second, is there a maximum length beyond which the modeling of a variable-depth liner as a uniform, smeared liner is invalid? Finally, would the previously reported benefits apply to ducts for which the impinging source structure is not constrained to plane waves, i.e., for which higher-order modes are present?

The current study begins to answer the first two of these questions, while retaining the constraint that the impinging source structure consists solely of plane waves, and the third question will be the focus of a follow-on study. A computational study (using the CHE code) is conducted to examine the effects of the lengths of the individual axial segments that comprise the variable-depth liner. The commercially available COMSOL propagation code has recently been added to the NASA suite of tools, and is used for a preliminary study to design optimized GFIT liner configurations. The results of this analysis are used to determine the suitability of the chosen metric for determining the 'optimum' liner configuration to achieve broadband noise suppression. Based on the results of these two initial studies, a constrained optimization procedure is used with the CDL and CHE codes to design a liner for the GFIT. The individual chambers that comprise the optimum liner are then rearranged to support optimum packaging. Two such configurations are built and tested in the GFIT.

Section II provides a description of the Grazing Flow Impedance Tube and the liner configurations considered in this study. The acoustic propagation models are briefly described in Section III, and results are provided in Section IV. Finally, conclusions relevant to this study are provided in Section V.

\section{Experimental Method}

\section{A. Grazing Flow Impedance Tube}

All liners considered in this study are designed to fit in the GFIT. The GFIT (see Fig. 1) has a cross-sectional geometry of 2.0" wide by 2.5 " high, and allows evaluation of acoustic liners with lengths from 2.0 to 24.0 ". The surface of the test liner forms a portion of the upper wall of the flow duct. Twelve acoustic drivers form an upstream (exhaust mode) source section. These drivers are typically used to generate tones (one frequency at a time) at source levels up to $150 \mathrm{~dB}$, over a frequency range of 0.4 to $3.0 \mathrm{kHz}$, at centerline Mach numbers up to 0.5. Fifty-three microphones are flush-mounted in the lower wall (opposite the liner) of the GFIT, and are used to measure the acoustic pressure field over the axial extent of $0 \leq x \leq L$ (see Fig. 2).

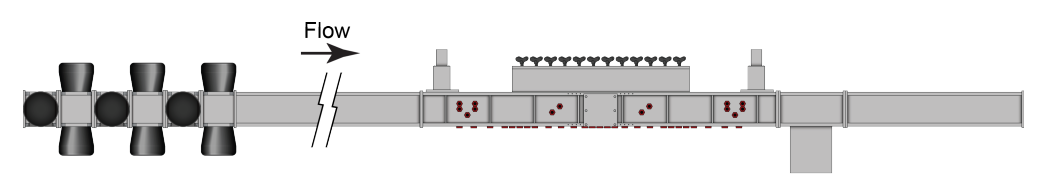

Figure 1: Sketch of Grazing Flow Impedance Tube (GFIT).

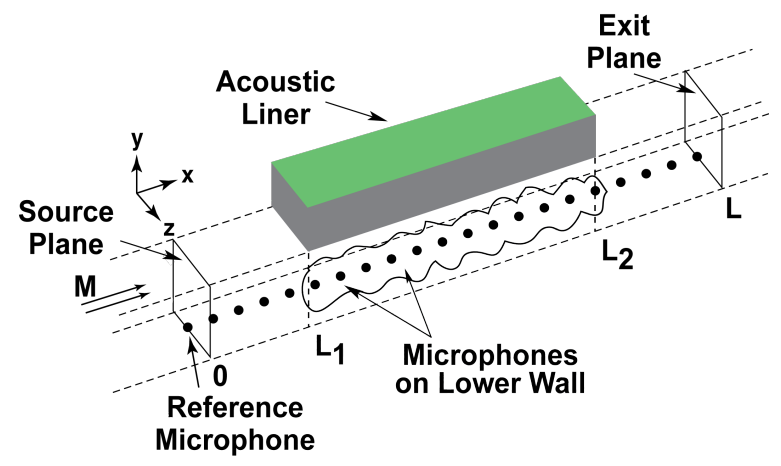

Figure 2: Sketch of Grazing Flow Impedance Tube test section. 


\section{B. Liner Configurations}

Three liner configurations, labeled as TL1, TL2, and TL3, are used in the computational part of this study. First, the TL1 configuration is used to determine whether there is a maximum length beyond which the modeling of a variable-depth liner as a uniform, smeared liner is invalid. Next, the TL2 configuration is used to determine the suitability of the chosen metric for selecting an optimum liner for broadband noise suppression. Finally, the TL3 configuration is used as a framework for the design of a liner optimized for broadband noise suppression in the GFIT.

The first configuration, TL1 (Fig. 3), has a 2.0" $\times 16.7$ " active surface, and contains one hundred and sixtyseven 2.0 "-wide by 0.1 "-long chambers that are assumed to be separated by infinitesimal-thickness partitions such that it can be treated as a locally-reacting liner. It employs a facesheet with a normalized resistance of 0.5 . The chamber depths increase linearly from 1.13 " at the leading edge to 3.38 " at the trailing edge of the active surface. Thus, the surface impedance, $\zeta_{i}$, of the $i^{\text {th }}$ chamber is given by

$$
\zeta_{i}=0.5-\cot \left(k d_{i}\right), \quad(i=1,167)
$$

where $k$ and $d_{i}$ represent the freespace wavenumber and depth of the $i^{\text {th }}$ chamber, respectively. The freespace wavenumber is given by

$$
k=\omega / c
$$

where $\omega=2 \pi f$ is the angular frequency and $f$ is the excitation frequency in Hz. (Note: An $e^{i \omega t}$ time convention is assumed. Also, all impedances in this paper are assumed normalized by $\rho c$, where $\rho$ and $c$ represent the density and sound speed of air, respectively.) This liner configuration is used to examine the modeling assumption of "smeared impedance". The liner can be modeled as 167 individual chambers or the chambers can be combined into axial groups containing arbitrary numbers of (smeared) chambers.

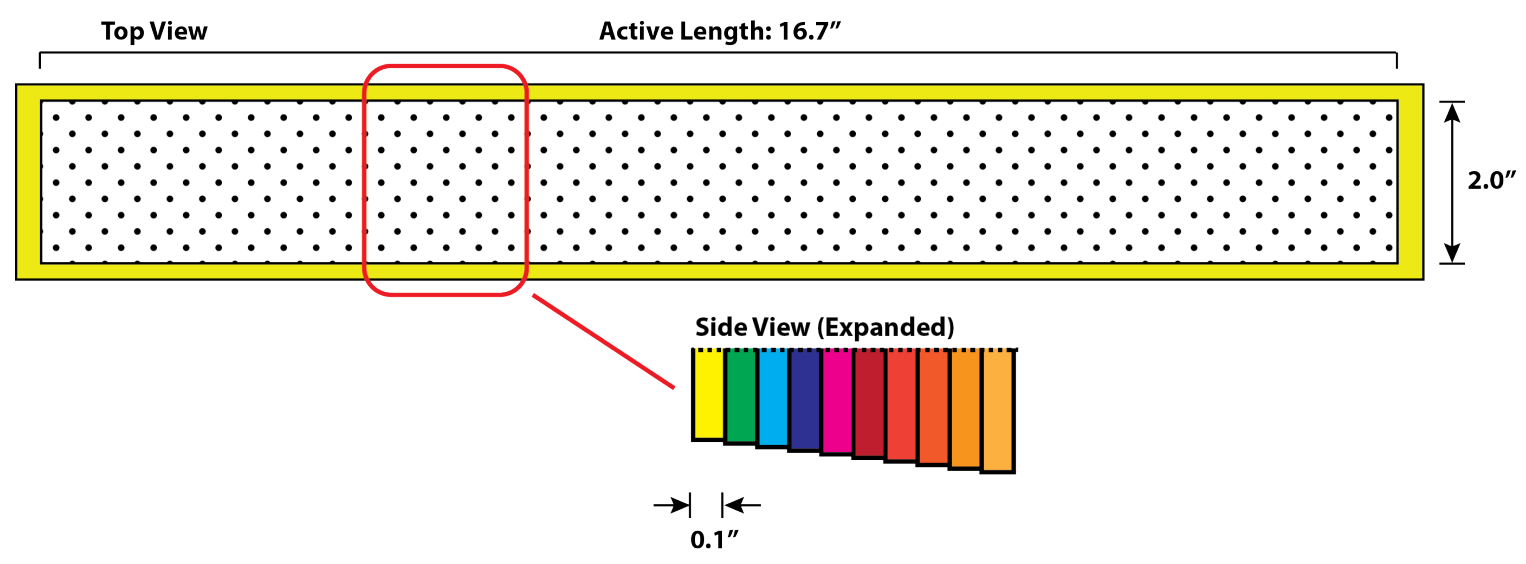

Figure 3: Sketch of liner geometry (TL1) used to determine acceptable 'smeared impedance' extent.

The second liner configuration, TL2 (Fig. 4), is used with the COMSOL code to design a liner based on a constrained optimization process. It contains an active surface of 2.0 " $\times 16.7$ ", and is comprised of eight 2.0 "-wide by 2.0 "-long chambers separated by 0.1 "-thick partitions. This optimization process allows each chamber to have a depth of $3.38 ", 2.25 ", 1.69 ", 1.35 "$, or 1.13 ", where the depths have been chosen to correspond to quarter-wavelengths at 1.0, 1.5, 2.0, 2.5, and $3.0 \mathrm{kHz}$. This liner has a wire mesh facesheet with a normalized resistance of 0.5 . This liner geometry was chosen to minimize the number of parameters needed in the optimization process, namely, the individual depths for each of the eight chambers. As a side note, it is perhaps worth noting that the dimensions of these chambers $(2.0$ " $\times 2.0$ ") are slightly larger than would be suggested by the results of the first evaluation with the TL1 configuration (see discussion below), 
but those results were not available when this portion of the study was initiated. Nevertheless, this was not critical for the purposes of this study.

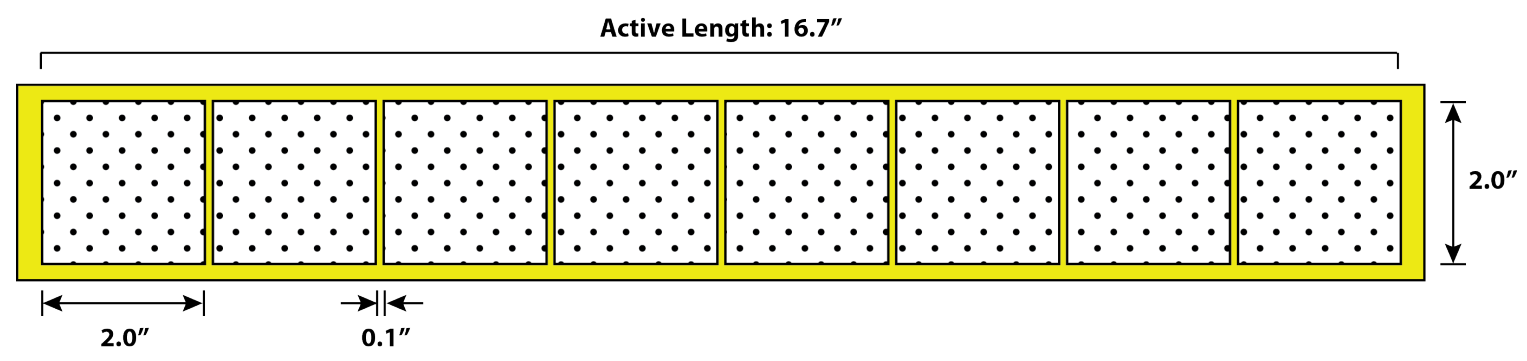

Figure 4: Sketch of liner geometry (TL2) used for initial constrained optimization analysis using COMSOL. Top View.

The final liner configuration, TL3 (Fig. 5), is used for the constrained optimization with the CDL and CHE codes. This configuration contains a 2.0 " $\times 16.7$ " active surface comprised of twenty-four 0.6 "-wide by 0.6 "-long chambers separated by 0.1 "-thick partitions in the lengthwise and spanwise directions. The facesheet for this liner is a wire mesh with a resistance of 0.5 , and the reactance of each chamber is given by $-\cot \left(k d_{i}\right)$. These parameters are chosen such that the final liner design can be built with the NASA Langley stereolithography apparatus. For the initial analysis with this liner configuration, $3 \times 3$ groups of chambers are assumed to be treated as a segment (i.e., each of the 9 chambers are identical). Thus, there are a total of eight segments, each of which is allowed to have any chamber depth between 1.0" and 4.0" in 0.1 " increments.

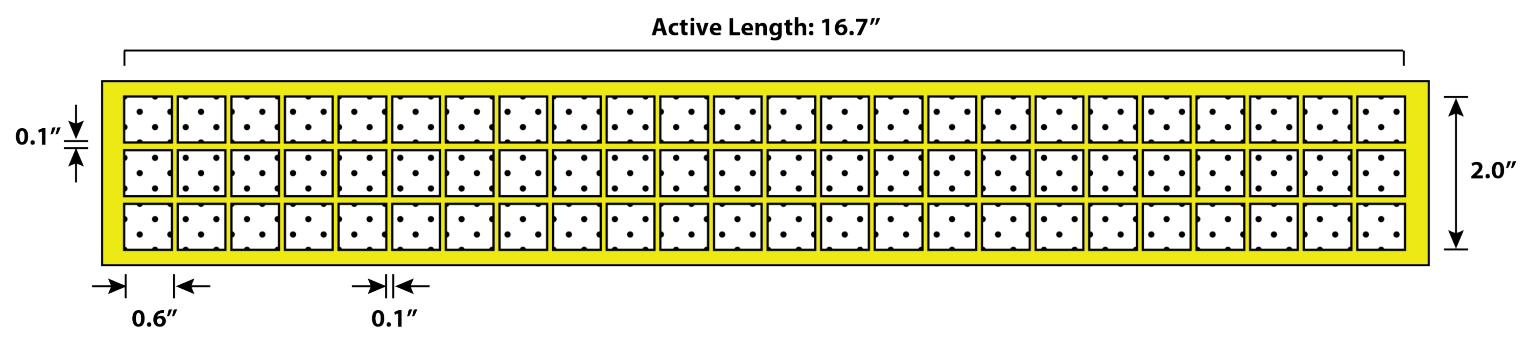

Figure 5: Sketch of liner geometry (TL3) used for full optimization using CHE and CDL. Top View.

\section{Duct Acoustic Propagation Codes}

Three duct acoustic propagation codes (COMSOL, ${ }^{7} \mathrm{CHE},{ }^{3}$ and $\mathrm{CDL}^{4}$ ) are used in this study. These codes are described in detail in the cited references, so only those details that are key to the current study are provided herein. Each of these codes solves the convected Helmholtz equation and, for the purposes of this study, employs a uniform flow profile. They also assume the liner to be installed on the upper wall (as in the GFIT) and allow the impedance to vary along the axial length of the liner. The COMSOL and CHE codes solve a partial differential equation on the acoustic pressure field. These codes include the effects of reflections at the leading $\left(x=L_{1}\right)$ and trailing $\left(x=L_{2}\right)$ edges of the liner and have the potential to capture reflections at the duct termination ( $x=L$, see Fig. 2). However, in the current study, an anechoic termination is used in both codes. The key difference in the COMSOL and CHE codes is in the order of the elements used in their respective finite element models. For this study, the COMSOL code used quadratic elements with the pressure variable as the nodal degree of freedom, whereas the CHE code used cubic elements with the pressure variable and its derivatives as the nodal degrees of freedom. Thus, the CHE code is expected to 
provide higher fidelity results for the same spatial resolution. The CDL propagation code utilizes a parabolic approximation to the convected Helmholtz equation with the acoustic potential as the unknown variable. The CDL code is a computationally efficient model that accounts for three-dimensional geometries. However, it neglects reflections due to impedance discontinuities at the leading and trailing edges of the liner. It is also constrained to cases for which the duct termination is anechoic.

\section{Results and Discussion}

This study can be divided into four components. First, a computational study is conducted with the CHE code and the TL1 liner configuration to determine whether there is a maximum length beyond which the modeling of a variable-depth liner as a uniform, smeared liner is invalid. Next, the COMSOL code is used to conduct a constrained optimization study based on the TL2 liner configuration. The results of this study are used to determine the suitability of the chosen metric for selecting an optimum liner for broadband noise suppression. Based on the results of the first two studies, a more detailed optimization procedure is used with the CDL and CHE codes to design a liner (TL3) for the GFIT. Because of the manner in which this optimization is performed, a much larger number of chamber depths can be considered. The individual chambers that comprise this optimum liner are then rearranged to support optimum packaging. This rearranging is first done at the segment level, then at the chamber level. The details of this rearranging exercise are provided later. Two liners based on these final designs are built and tested (at Mach 0.0 only) in the GFIT. Finally, results of these tests are compared to CHE predictions to validate the liner design process.

\section{A. Evaluation of smeared impedance assumption}

It has previously ${ }^{8}$ been assumed that spatial variation, whether axial or spanwise, in surface impedance can acceptably be represented with the corresponding 'smeared' impedance in duct acoustic propagation codes as long as the spatial extent is no greater than one-eighth of a wavelength at the highest frequency of interest. For this portion of the current study, the highest frequency considered is $2.0 \mathrm{kHz}$. The wavelength, $\lambda$, is given by

$$
\lambda=c /[f(1 \pm M)]
$$

where $M$ represents the Mach number of the mean flow in the duct. Thus, the wavelength at $2.0 \mathrm{kHz}$ is 6.75 " in the absence of mean flow. For Mach numbers of 0.3 and 0.5 (the two flow speeds used in this study), this wavelength grows due to the convective effects of the mean flow to lengths of 9.64" and 13.50 ", respectively, for forward-propagating waves and reduces to lengths of 5.19 " and $4.5 "$, respectively, for backward-propagating waves. Thus, the aforementioned $\lambda / 8$ assumption would indicate that a smeared impedance can be successfully used to represent axial impedance variability that occurs over a length no greater than 0.84 in, 0.65 in, and 0.56 in at Mach numbers of 0.0, 0.3, and 0.5 (based on the smaller of the two choices for the flow conditions), respectively.

As mentioned earlier, the TL1 liner configuration is comprised of one hundred and sixty-seven 2.0"-wide by 0.1 "'-long chambers, and has a wire mesh facesheet with a normalized resistance of 0.5 . The chamber depths increase linearly from 1.13 " at the leading edge to 3.38 " at the trailing edge. The CHE code is used to compute the axial sound pressure level profile, $\operatorname{SPL}(x)$, for the full, 167-chamber liner along the lower wall (opposite of liner) of the duct. This SPL profile is the baseline against which the successive smeared impedance calculations are compared. These 167 chambers are then combined into multi-chamber segments, where the smeared impedance for a segment is determined as follows. First, the admittance of the $i^{\text {th }}$ chamber, $\beta_{i}$, is computed as

$$
\beta_{i}=1 / \zeta_{i}
$$

where $\zeta_{i}$ represents the impedance of the $i^{\text {th }}$ chamber. The smeared (average) admittance over the segment, $\beta_{s}$, is given by 


$$
\beta_{s}=\frac{1}{N} \sum_{i=1}^{N} \beta_{i}
$$

where $N$ represents the number of chambers included in the segment. Finally, the smeared impedance for the segment, $\zeta_{s}$, is given by

$$
\zeta_{s}=1 / \beta_{s}
$$

Results were computed for segments containing 10 to 40 chambers, in 5-chamber increments, corresponding to segment lengths of 1.0" to 4.0". These computations were performed for mean flow Mach numbers of 0.0, 0.3 , and 0.5 .

For example, if 25-chamber segments are chosen, this means that a smeared impedance is computed for the first 25 chambers (length of 2.5"), labeled as segment 1. A second smeared impedance is computed for the next 25 chambers, labeled as segment 2, etc. Since each segment has a length of $2.5 \%$, there are six full segments (total of 15"). The remaining 17 chambers (length of 1.7") form the final segment. Thus, there are a total of seven segments, with corresponding smeared impedances, that comprise the '25-chamber' liner. This configuration is used as input to the CHE code, and the resultant SPL profile along the lower wall of the duct is compared against that achieved with the baseline configuration (167 independent chambers).

If the $\lambda / 8$ assumption holds, the SPL profile for each of these segment lengths should be noticeably different from the profile for the full, 167-chamber liner, since the shortest segment length of 1.0" (10chamber segments) is longer than the $\lambda / 8$ values indicated above. The results shown in Fig. 6 demonstrate that this is not true. Not shown in the figure, the predicted SPL profiles for liners consisting of 10-, 15-, and 20-chamber segments (i.e., uniform impedance for 1.0", 1.5", and 2.0") are nearly identical to the predicted SPL profile with 1-chamber segments (original, full liner). As the segment length is increased above 2.0", the SPL profiles begin to deviate from the baseline profile. Figure $6 \mathrm{~b}$ provides a detailed review as the segment length increases from 2.5" (25 chambers) to 4.0" (40 chambers). At 25 chambers, the maximum deviation from the original prediction is seen to be less than $0.5 \mathrm{~dB}$. This increases with increasing segment length, with a maximum deviation of less than $2.0 \mathrm{~dB}$ for the largest segment length (4.0"). These results indicate a segment length of at least 2.5", slightly larger than one-third of a wavelength at $2.0 \mathrm{kHz}$, is acceptable. This is much larger than previously assumed.

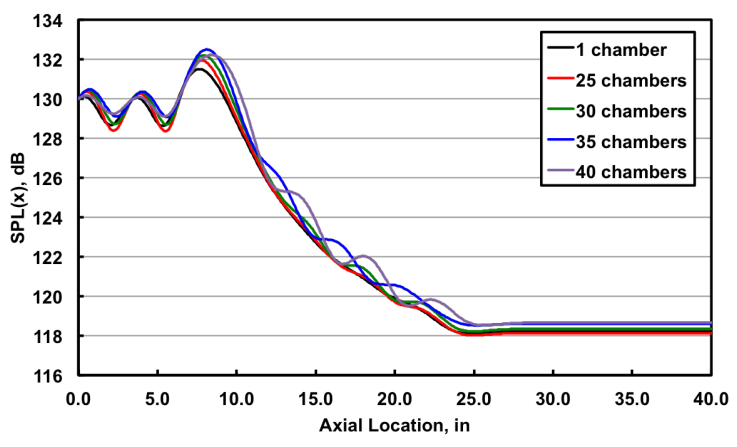

(a) Sound pressure level profiles.

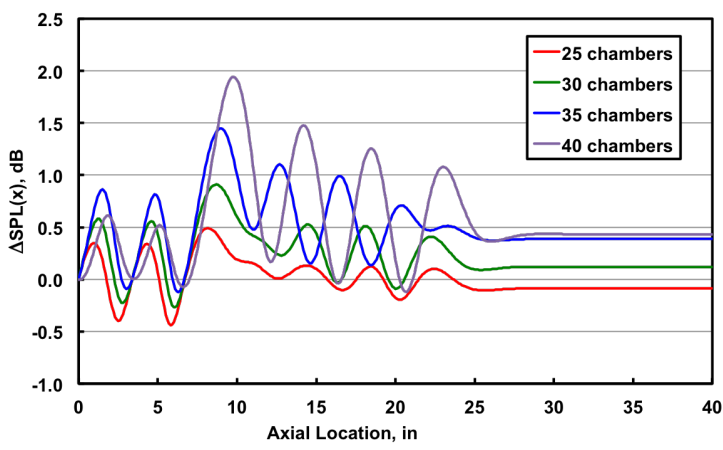

(b) Comparison of SPL for N-chamber segment relative to original liner.

Figure 6: Comparison of predicted sound pressure level profiles 1-, 25-, 30-, 35-, and 40-chamber segments; Mach 0.0; $2.0 \mathrm{kHz}$.

Similar results are observed for predictions computed at Mach numbers of 0.3 and 0.5 . In fact, those results suggest that $\lambda / 3$ is conservative, i.e., it may be acceptable to 'smear' the impedance over a longer segment length. Regardless, for the purposes of this study, the $\lambda / 3$ limit will be observed. 


\section{B. Optimization metric}

The COMSOL code, which allows for convenient parametric studies, was used to conduct an initial constrained optimization liner design. The goal of this optimization study was to evaluate different metrics that could be used to assess the 'value' of different liner configurations. This code allows the user to sort through each potential combination of chamber depths to find an optimum configuration, and was therefore convenient for this metric evaluation. As mentioned in Section II.B, an 8-chamber liner (TL2, see Fig. 4a) was used for this optimization study.

A number of metrics were considered, but the discussion herein is limited to the one deemed most applicable to this study. The goal of this study was to determine the optimum set of chamber depths to achieve a liner that would provide maximum attenuation over the frequency range of 1.0 to $3.0 \mathrm{kHz}$. The 16.7"-long liner was assumed to be placed in the GFIT with the leading edge of the active surface positioned at $x=L_{1}$ (see Fig. 2), with the source SPL set to $130 \mathrm{~dB}$ (re $20 \mu \mathrm{Pa}$ ). Thus, the attenuation, $A_{f}$, at an individual frequency is simply computed as

$$
A_{f}=130-\mathrm{SPL}_{t}
$$

where $\mathrm{SPL}_{t}$ represents the sound pressure level $(\mathrm{dB})$ at the termination $(x=L)$. In order to minimize the computational resource requirements, only five chamber depths were considered (3.38", 2.25", 1.69", 1.35", and 1.13") to correspond to quarter-wavelengths at 1.0, 1.5, 2.0, 2.5, and $3.0 \mathrm{kHz}$.

The basic optimization process is described as follows. First, the COMSOL code selects chamber depths for each of the eight chambers. The sound pressure level axial profile, $\operatorname{SPL}(x)$, is then computed at frequencies of 1.0 to $3.0 \mathrm{kHz}$ in $0.25 \mathrm{kHz}$ increments (total of nine frequencies), from which the respective termination sound pressure levels, $\mathrm{SPL}_{t}$, are determined. Each of these levels is converted to the corresponding RMS acoustic pressure, $P_{t}(\mathrm{~Pa})$, as

$$
P_{t}=\left(20 \times 10^{-6}\right) 10^{\mathrm{SPL}_{t} / 20}
$$

Finally, the sum of the squares of these nine acoustic pressures is used as the objective function, $P_{\min }^{2}$, to be minimized, i.e.,

$$
P_{\text {min }}^{2}=\sum_{j=1}^{9} P_{t, j}^{2}
$$

Figure 7 provides results for the three best liner geometries predicted via this constrained optimization. Figure $7 \mathrm{a}$ provides the depths of the eight chambers, and Fig. 7b provides the termination sound pressure levels $\left(S P L_{t}\right)$ for each configuration. This optimization, conducted for the no-flow condition, indicates that a minimum of $10 \mathrm{~dB}$ suppression is achievable across the full frequency range, which confirms the suitability of the chosen optimization metric. Also, since this optimization was constrained to allow for only five chamber depths, these results are assumed to represent a nominal lower limit for what could be achieved with a greater number of chamber depths.

\section{Constrained optimization for liner design}

As mentioned above, the COMSOL code allowed the liner parameters (in this case, the individual chamber depths) to be varied over a range of values to determine an optimum configuration. However, in order to minimize computational resource requirements, only five chamber depths could be included. For the final liner design, a much finer resolution is desired. For this design, the desired range of chamber depths is 1.0" to $3.5 "$.

The basic liner configuration (TL3), described earlier in Section II.B, consists of an array of $3 \times 24$ chambers. Each chamber has a 0.6 " $\times 0.6$ " cross-section, and is separated from adjacent chambers via 0.1"-thick partitions in the lengthwise and spanwise directions. Groups (segments) of $3 \times 3$ chambers are assumed to have identical depths and, therefore, identical surface impedances. For this portion of the study, the target 


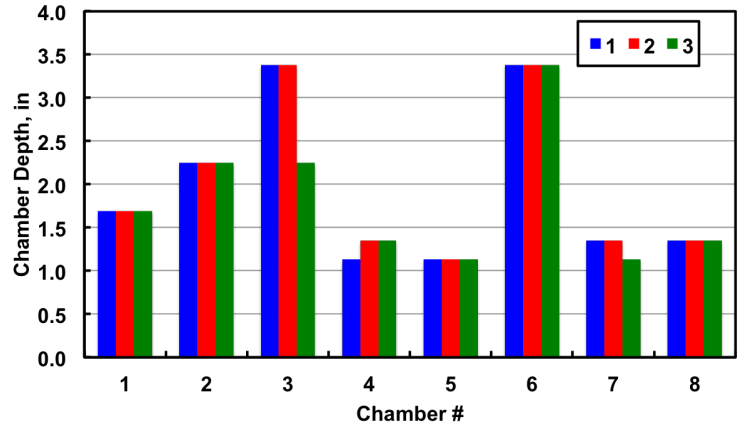

(a) Chamber depths (in).

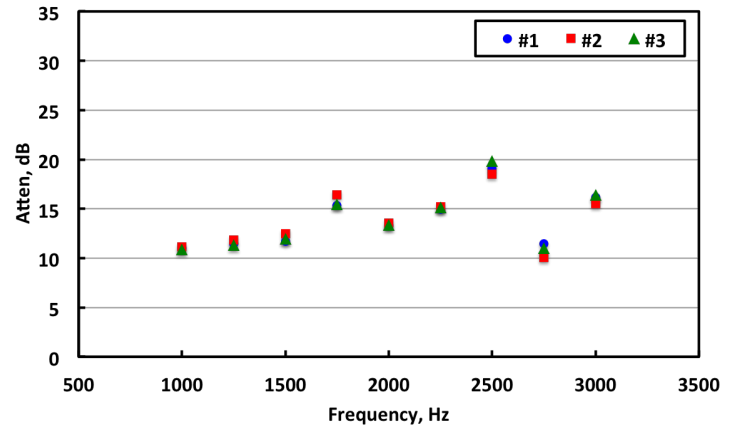

(b) Predicted attenuation spectra $[A=S P L(0)-S P L(L)]$.

Figure 7: Comparison of results for the three best configurations based on optimizations conducted with COMSOL; Mach 0.0.

frequency range is from $1000 \mathrm{~Hz}$ up to the frequency at which the first higher-order mode is cut on (i.e., this is the frequency range used for calculation of the objective function). For the purpose of comparisons, results are computed at frequencies of 1000 to $2600 \mathrm{~Hz}$ in $200 \mathrm{~Hz}$ increments.

None of the three propagation codes (COMSOL, CDL, CHE) has the built-in flexibility to support liner optimization that allows for a large number of liner depths in a timely manner. However, the CDL and CHE codes are well suited for combination with optimizers developed using the Python programming language. ${ }^{9}$ This allows access to the full set of optimization tools available in the optimization package of the Scipy ${ }^{10}$ library. While a number of constrained optimization methods are available, the possibility of many local optima led to the use of two global optimizers based on the Basin-Hopping and Differential Evolution algorithms. Both are stochastic in nature and attempt to find the global minimum of a multivariate function. The Basin-Hopping algorithm is iterative with each cycle consisting of a random perturbation of the coordinates, a local minimization, and acceptance/rejection of the new coordinates based on the objective function value. In this case, the local minimization is carried out using a standard gradient based method. The Differential Evolution algorithm, conversely, does not use gradient methods. This algorithm begins with a candidate population and with each iteration the algorithm mutates each candidate solution by mixing with other candidate solutions to create a trial candidate. The fitness of each trial candidate is assessed in relation to the objective function and the calculations continue until prescribed tolerances or maximum iterations are reached.

The CDL code is used with each of these optimizers to determine the respective depths of the eight segments (groups of $3 \times 3$ chambers). The selected segment depths are then used as input to the CHE (higher fidelity) code to predict SPL and phase profiles (only SPL used for current study), and to compute final values of the objective function. First, this optimization process is used to determine the optimum configuration for the Mach 0.0 condition (i.e., the better of the configurations selected with the two optimizers). The process is repeated for the Mach 0.3 condition. As might be expected, the two liner configurations are not the same. Therefore, in order to down-select to a single configuration, the first configuration is reanalyzed for the Mach 0.3 condition and the second configuration is reanalyzed for the Mach 0.0 condition (i.e., each liner is evaluated at its off-design condition). These results are then used to select one of the configurations for further review.

Figure 8 provides attenuation spectra at the two flow conditions for the liner that was optimized for the Mach 0.0 condition. This configuration was selected based on results achieved with the Differential Evolution optimizer. At Mach 0.0, the first higher-order mode cuts on in the GFIT above $2600 \mathrm{~Hz}$. Thus, the entire frequency range (1000 to $2600 \mathrm{~Hz}$ ) is used in the optimization. This liner provides approximately 5 to $10 \mathrm{~dB}$ more attenuation at each frequency for the Mach 0.0 condition than for the Mach 0.3 condition. There is significant variation in the eight segment depths, ranging from 1.00" to 3.14 ". Indeed, two of the them (third and seventh segments) have depths of 1.0", the minimum allowed. It should be noted that this design space appears to have numerous local optima. Thus, the ability of the optimizer to find a global optimum is clearly dependent on the appropriate selection of control parameters (e.g., tolerances). Thus, it is entirely 


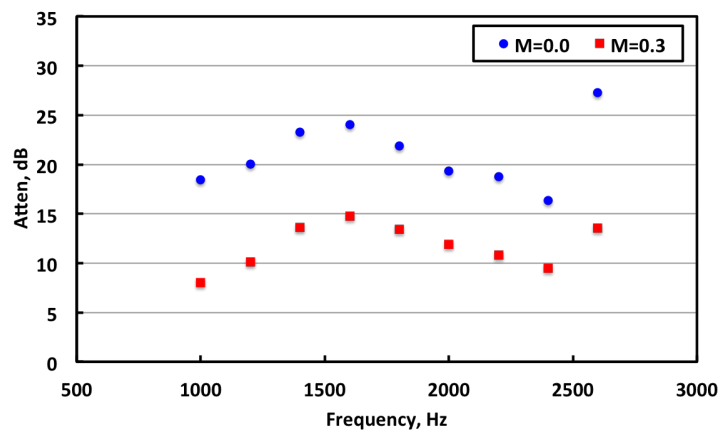

Figure 8: Predicted attenuation spectra, optimized for Mach 0.0 condition using the Differential Evolution optimizer. Segment depths of 1.79", 2.12", 1.00", 3.14", 1.17", 3.13", 1.00", and 1.72", respectively.

possible that there exists an even better configuration within the design space. Nevertheless, the current liner provides significant broadband attenuation, which is the goal of this study.

Corresponding attenuation spectra are provided in Figure 9 for the liner optimized for the Mach 0.3 condition. Although this configuration was selected based on results achieved with the Basin-Hopping optimizer, the results achieved with the Differential Evolution optimizer were quite similar. In general, this was observed for all of the configurations considered. At this Mach number, the first higher-order mode cuts on in the GFIT above $2400 \mathrm{~Hz}$. Thus, the optimization is constrained to the frequency range of 1000 to $2400 \mathrm{~Hz}$. The segment depths for this liner range from 1.14" to 3.50". Whereas the liner optimized at Mach 0.0 had two segments at the minimum depth, this liner has none. However, it has one segment at the maximum depth of 3.50 ".

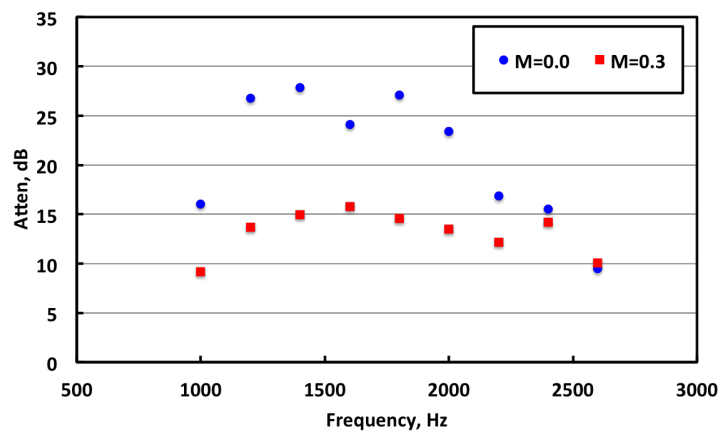

Figure 9: Predicted attenuation spectra for Configuration C1, optimized for Mach 0.3 condition using the Basin-Hopping optimizer. Segment depths of 1.72", 3.50", 1.14", 1.19", 1.46", 2.06", 2.32", and 2.24", respectively.

A comparison of Figures 8 and 9 indicates both liners provide similar attenuation in spite of the fact that they were optimized for different test conditions and have different arrangements of segment depths. By comparing the respective objective functions at both the design and off-design conditions, the latter configuration is selected as the best overall configuration based on the metric provided in Equation (9). This liner is therefore the one selected for more detailed analysis. For convenience, this configuration is labeled as 'C1.' 


\section{Reordering results}

Previous studies have suggested that it is possible to reorder the segments without sacrificing much of the broadband attenuation achieved with the optimized liner configuration. ${ }^{2}$ It is of interest to take advantage of this concept to achieve a liner configuration that is more suitable to fabrication using bent chambers in order to reduce the overall depth (thickness) of the liner.

In order to explore this idea, the chambers within the successive segments are redistributed in the following manner. Chambers in the first six $3 \times 1$ rows (chambers in spanwise direction have constant depth) alternate between depths of 1.72" and 3.50" and cover the same axial extent as was initially covered with the first two segments. Chambers in the next six $3 \times 1$ rows alternate between depths of 1.14 " and 1.19". The next six rows use depths of 1.46" and 2.04", and the last six rows use depths of 2.32" and 2.24". This configuration is labeled as 'C2.' The predicted attenuation spectra for this liner configuration (Fig. 10) are quite similar to those for the optimized configuration (Fig. 9). This design places the longest and shortest chambers (and next longest and next shortest, etc.) in adjacent positions.

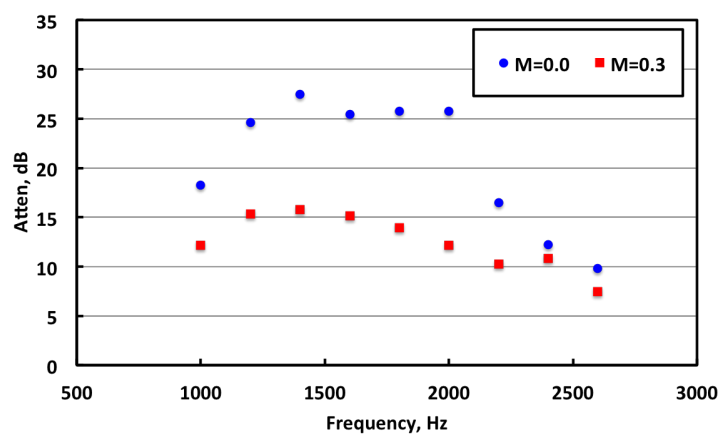

Figure 10: Predicted attenuation spectra for configuration C2. First set of six spanwise rows contains alternating chamber depths of 1.72" and 3.50". Second set uses depths of 1.14" and 1.19". Third set uses depths of 1.46 " and 2.06". Final set uses depths of 2.32" and 2.24".

\section{E. Comparison of predicted and measured results}

The two configurations described above $(\mathrm{C} 1$ and $\mathrm{C} 2)$ were fabricated using additive manufacturing. Each of them correspond to configuration TL3 (Fig. 5), and the only distinction is the distribution of chamber depths as described in the previous section. Both liners were covered with the same 270 MKS Rayls wire mesh facesheet. Acoustic pressure data were acquired with each sample mounted in the GFIT, at frequencies of 600 to $3000 \mathrm{~Hz}$, and at Mach 0.0. The following figures provide comparisons of the predicted and measured attenuation spectra.

Figure 11 provides this comparison for the $\mathrm{C} 1$ liner. While there are differences, especially near the frequency of peak attenuation $(1600 \mathrm{~Hz})$, the comparison is quite favorable. Corresponding results for the C2 liner, shown in Fig. 12, confirm that the acoustic propagation code (CHE) does an excellent job of predicting the attenuation when this type of liner is mounted in the GFIT.

Finally, it is perhaps of interest to note the nature of the measured SPL profiles. Figure 13 provides these profiles for frequencies of 1000, 1400, 1800, and $2200 \mathrm{~Hz}$. These results are generally representative of the results achieved throughout the entire frequency range. There is clear evidence of a standing wave pattern at the downstream end of the test section, which is stronger for some frequencies than for others. It should be noted that the termination impedance of the GFIT is not completely anechoic, as is assumed in the predictions. These results demonstrate that the downstream termination impedance has a strong frequency dependence. Thus, differences between the predicted and measured attenuation spectra presented above (Figs. 11 and 12) are to be expected. 


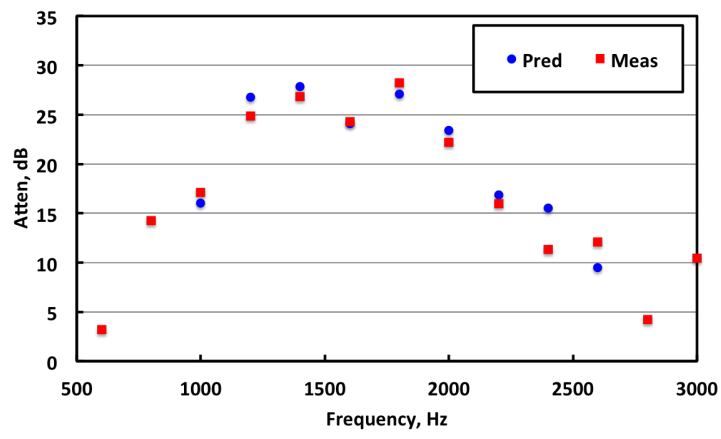

Figure 11: Comparison of predicted and measured attenuation for C1 liner; Mach 0.0.

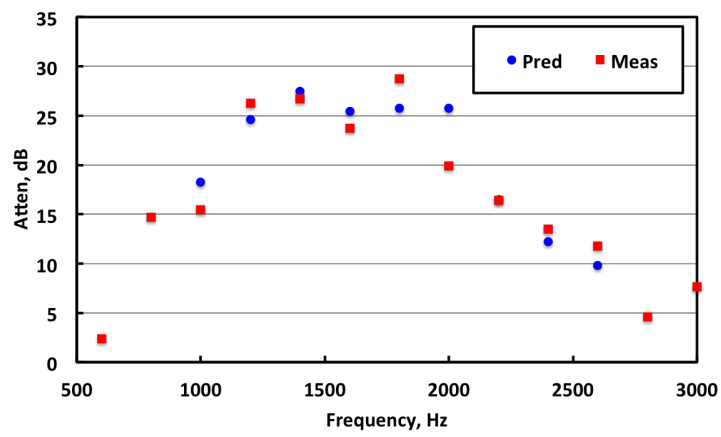

Figure 12: Comparison of predicted and measured attenuation for C2 liner; Mach 0.0.

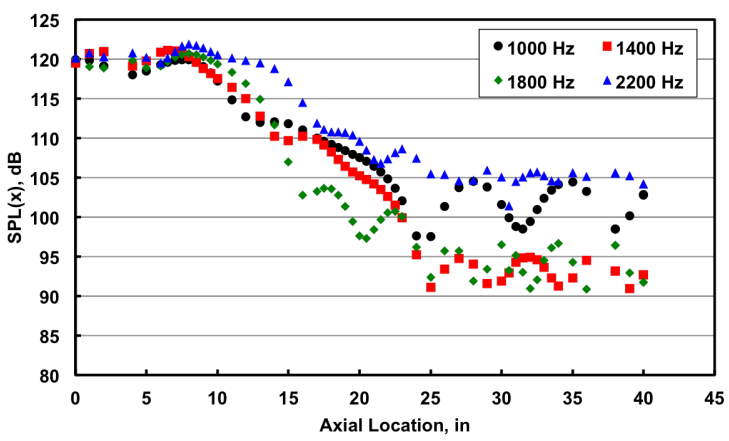

Figure 13: Measured sound pressure level profiles for C2 liner; Mach 0.0.

\section{Concluding Remarks}

This paper has explored multiple effects of variable-depth liner configurations with the use of three acoustic propagation codes, CHE, COMSOL, and CDL, applied to the geometry of the NASA Langley Grazing Flow Impedance Tube (GFIT). Each code employs a distinct approach to solve the convected Helmholtz equation. The initial study used CHE to determine the spatial extent over which a variable-impedance can 
acceptably be treated as a uniform, 'smeared' impedance. The results suggest that the maximum spatial extent is approximately one-third of the wavelength of the highest frequency being considered.

The COMSOL code was used to conduct a constrained (limited number of chamber depths) optimization study to design an initial variable-depth liner for the GFIT. Perhaps more importantly, this analysis was used to select an optimization metric and to gain insight into how much attenuation can be achieved in the GFIT with a variable-depth liner.

Next, the CDL propagation code was combined with two optimizers (Basin-Hopping and Differential Evolution) to design a liner for use in the GFIT. These optimizers allowed a much finer resolution of chamber depths. After each optimization was concluded, the CHE propagation code (higher-fidelity code) was used to compute the final value of the optimization metric. One liner was designed based on optimizations (with each optimizer) at the Mach 0.0 test condition, and a second was designed for the Mach 0.3 test condition. Each liner was then evaluated at the alternate Mach number (i.e., the off-design condition) to determine which liner provided the best combined (Mach 0.0 and 0.3 ) attenuations over the frequency range of interest (400 to $2600 \mathrm{~Hz}$ ). The one optimized for the Mach 0.3 condition provided slightly better results.

One of the eventual goals of the current research is to design variable-depth liners with bent chambers to take advantage of packaging optimization. To this end, a liner was designed in which the rows with different depths were interleaved. Thus, the first chamber in the axial dimension was at a depth corresponding to the depth of the first segment in the optimized liner, while the second chamber was at a depth corresponding to the depth of the second segment in the optimized liner. This pattern was repeated, such that the depths of the first six chambers in the axial dimension alternated between these two settings (chambers in the spanwise dimension always maintained a constant depth). The next six chambers used the next two available depths (next longest and next shortest), and this approach was repeated through the length of the liner. Results from CHE indicate this liner provides very similar attenuation to the original configuration.

Each of these liners was fabricated using additive manufacturing, and was tested in the GFIT at Mach 0.0. Comparisons between predicted (using CHE) and measured attenuations are excellent across the entire frequency range. These results clearly suggest that reordering of the chambers is acceptable, such that they can be arranged in an order suitable for repackaging with bent chambers to reduce the overall liner depth. Plans are underway to conduct tests at Mach 0.3 for further comparison with the predicted attenuations, and to build another liner to confirm that similar results can be achieved with bent chambers.

\section{Acknowledgments}

The authors wish to express sincere appreciation to Carol Harrison, Martha Brown, and Brian Howerton for their efforts in the acquisition of the GFIT data. The Advanced Air Transportation Technologies Project of NASA's Advanced Air Vehicle Program funded this work.

\section{References}

\footnotetext{
${ }^{1}$ Jones, M. G., Watson, W. R., Nark, D. M., and Howerton, B. M., "Evaluation of a Variable-Impedance Ceramic Matrix Composite Acoustic Liner," AIAA Paper 2014-3352, June 2014.

${ }^{2}$ Jones, M. G., Watson, W. R., Nark, D. M., and Howerton, B. M., "Evaluation of Variable-Impedance Liner Configurations for Increased Broadband Noise," AIAA Paper 2015-2697, June 2015.

${ }^{3}$ Watson, W. R., Jones, M. G., and Parrott, T. L., "Validation of an Impedance Eduction Method in Flow," AIAA Journal, Vol. 37, No. 7, July 1999, pp. 818-824.

${ }^{4}$ Nark, D. M. and Jones, M. G., "Broadband Liner Optimization for the Source Diagnostic Test Fan," AIAA Paper 2012-2195, June 2012.

${ }^{5}$ Howerton, B. M. and Parrott, T. L., "Validation of an Acoustic Impedance Prediction Model for Skewed Resonators," AIAA Paper 2009-3143, May 2009.

${ }^{6}$ Howerton, B. M., Jones, M. G., and Buckley, J. L., "Development and Validation of an Interactive Liner Design and Impedance Modeling Tool," AIAA Paper 2012-2197, June 2012.

7"COMSOL Acoustic Module User's Guide," v5.1, 2015.

${ }^{8}$ Parrott, T. L. and Jones, M. G., "Parallel-Element Liner Impedances for Improved Absorption of Broadband Sound in Ducts," Noise Control Engineering Journal, Vol. 43, No. 6, November - December, 1995.

9"URL: http://www.python.org," [cited 25 March 2016].

${ }^{10}$ Jones, E., Oliphant, T., Peterson, P., et al., "SciPy: Open source scientific tools for Python," URL: http://www.scipy.org/, 2001-.
} 